\title{
Epitaxial Growth of Nanorod Meshes from Luminescent Organic Cocrystals via Crystal Transformation
}

Yanqiu Sun ${ }^{\dagger}$ Yilong Lei $^{*}{ }^{*}$ Wenping Hu, ${ }^{*}$ and Wai-Yeung Wong ${ }^{* \dagger}$

${ }^{\dagger}$ Department of Applied Biology and Chemical Technology, The Hong Kong Polytechnic University (PolyU), Hung Hom, Hong Kong, P. R. China; PolyU Shenzhen Research Institute, Shenzhen, 518057, P. R. China

${ }^{*}$ Department of Chemistry, School of Science, Tianjin University, Tianjin, 300072, P. R. China

\section{Contents}

1. Experimental methods

2. Figure S1. The simulated morphology of BP crystal based on growth morphology method.

3. Table S1. Summary of crystallographic data of BP crystal, BP-1,3-DTFB cocrystal and BP-1,4DTFB cocrystal.

4. Figure S2. Molecular packing modes of BP crystal perpendicular to the (a) $a-$, (b) $b$-, and (c) $c$-axis. (d) Intermolecular distances were measured in BP crystal.

5. Figure S3. Molecular packing modes of BP-1,3-DTFB cocrystal perpendicular to the (a) $a$-, (b) $b$-, and (c) $c$-axis. (d) Intermolecular distances were measured in BP-1,3-DTFB cocrystal.

6. Figure S4. Molecular packing modes of BP-1,4-DTFB cocrystal perpendicular to the (a) $a$-, (b) $b$-, and (c) $c$-axis. (d) Intermolecular distances were measured in BP-1,4-DTFB cocrystal.

7. Figure S5. The simulated morphology of BP-1,3-DTFB cocrystal based on growth morphology method.

8. Figure S6. (a) FM and (b) SEM images of small-sized BP sheets with a width of around $50 \mu \mathrm{m}$. (c) FM and (d) SEM images small-sized BP-1,3-DTFB nanorod meshes.

9. Figure S7. FM images of partially transformed BP microsheets upon (a) immersing them into 1,3DTFB solution in IPA with low concentration $\left(\mathrm{C}_{\mathrm{DTFB}}=50 \mathrm{mM}\right)$ or $(\mathrm{b})$ rapid evaporation of the suspension of BP microsheets dispersed in 1,3-DTFB solution in IPA $\left(\mathrm{C}_{\mathrm{DTFB}}=100 \mathrm{mM}\right)$.

10. Figure S8. (a) FM and (b) SEM images of multilayer BP-1,3-DTFB nanorod meshes.

11. Figure S9. (a) PL spectra of BP-based assemblies involving BP microsheets, BP-1,4-DTFB microwires, and BP-1,4-DTFB nanorod meshes. (b) The corresponding PXRD patterns.

12. Figure S10. FM images of (a) BP-octafluoronaphthalene (BP-OFN) cocrystal microwires, (b, c) partially transformed BP-OFN nanorod meshes, and (d) SEM image of a single partially transformed BP-OFN nanorod mesh. 
13. Figure S11. FM images of (a) BP-TCNB cocrystal microwires, (b, c) BP-TCNB nanorod meshes, and (d) SEM image of a single BP-TCNB nanorod mesh.

14. Figure S12. The simulated morphology of BP-1,4-DTFB cocrystal based on growth morphology method.

15. Table S2. Calculated attachment energies of different crystal facets of BP crystal.

16. Table S3. Calculated attachment energies of different crystal facets of BP-1,3-DTFB crystal.

17. Table S4. Calculated attachment energies of different crystal facets of BP-1,4-DTFB cocrystal. 


\section{Experimental Methods}

Materials: Benzoperylene (BP, > 97\%), 1,3-dicyanotetrafluorobenzene (1,3-DTFB, > 98\%), 1,4dicyanotetrafluorobenzene $(1,4-\mathrm{DTFB},>98 \%)$, 1,2,4,5-tetracyanobenzene $(\mathrm{TCNB},>98 \%)$ and octafluoronaphthalene (OFN, > 97\%) were purchased from TCI. Tetrahydrofuran (THF, HPLC), toluene (A.R.), and isopropyl alcohol (IPA, A.R.) were purchased from Beijing Chemical Ltd. China. All of the chemicals were directly used without further purification.

Synthesis: 2D BP microsheets with a large- $(\sim 150 \mu \mathrm{m})$ or small-sized width $(\sim 50 \mu \mathrm{m})$ were obtained by slow or fast evaporation of one drop of $\mathrm{BP}$ solution in toluene $\left(\mathrm{C}_{\mathrm{BP}}=30 \mathrm{mM}\right)$ at room temperature. Moreover, 1D BP-1,3-DTFB microwires were also synthesized by slow evaporation of a drop of $\mathrm{BP} / 1,3-\mathrm{DTFB}$ solution in THF with 1:1 molar ratio $\left(\mathrm{C}_{\mathrm{BP}}=\mathrm{C}_{\mathrm{DTFB}}=50 \mathrm{mM}\right)$ on a quartz substrate. Unexpectedly, BP-1,3-DTFB nanorod meshes were formed by immersion of the BP nanosheets into the 1,3-DTFB solution in IPA $\left(\mathrm{C}_{\mathrm{DTFB}}=100 \mathrm{mM}\right)$ and then slow evaporation of the suspension at room temperature. Similarly, 1D BP-1,4-DTFB microwires were also synthesized by slow evaporation of a drop of $\mathrm{BP} / 1,4-\mathrm{DTFB}$ solution in THF with 1:1 molar ratio $\left(\mathrm{C}_{\mathrm{BP}}=\mathrm{C}_{\mathrm{DTFB}}=50 \mathrm{mM}\right)$ on a quartz substrate. Also, BP-1,4-DTFB nanorod meshes were formed by immersion of the BP nanosheets into the 1,4-DTFB solution in IPA $\left(\mathrm{C}_{\mathrm{DTFB}}=100 \mathrm{mM}\right)$ and then slow evaporation of the suspension at room temperature. BP-octafluoronaphthalene (BP-OFN) microwires, partially transformed BP-OFN nanorod meshes, BP-1,2,4,5-tetracyanobenzene (BP-TCNB) microwires, and BP-TCNB nanorod meshes were also formed following similar preparation procedures to BP-DTFB nanorod meshes except that electron acceptors were replaced with OFN and TCNB.

Characterization: The morphologies and sizes of the samples were examined using field-emission scanning electron microscopy (FESEM, FEI Quanta $200 \mathrm{~F}$ ) at an acceleration voltage of $5 \mathrm{kV}$. Prior to analysis, the samples were coated with a thin gold layer using an Edwards Sputter Coater. TEM images were obtained using a Philips CM 200 electron microscope operated at $80 \mathrm{kV}$. One drop of the asprepared colloidal dispersion was deposited on a carbon-coated copper grid, and dried under high vacuum. The X-ray diffraction (XRD) patterns were measured by a D/max 2400 X-ray diffractometer with $\mathrm{Cu} \mathrm{K \alpha}$ radiation $(\lambda=1.54050 \AA)$ operated in the $2 \theta$ range from 5 to $40^{\circ}$, by using the samples filtered on the surface of a quartz substrate. The crystallographic data of BP-1,3-DTFB and BP-1,4- 
DTFB cocrystals was collected on a single crystal X-ray diffractometer (X-Pert, PANalytical, Netherlands) with $\mathrm{Cu} \mathrm{K} \alpha$ radiation $(40 \mathrm{kV}, 30 \mathrm{~mA})$. The photoluminescence (PL) spectra and solid-state PL quantum yields (PLQYs) of BP-based assemblies were measured on a HORIBA JOBTN YVON FLUOROMAX-4 spectrofluorimeter with a slit width of $1 \mathrm{~nm}$. All the samples were deposited on the surface of a quartz substrate. The fluorescence microscopy images were obtained using a Leica DMRBE fluorescence microscopy with a spot-enhanced charged couple device (CCD, Diagnostic Instrument, Inc.). The growth morphologies of BP, BP-1,3-DTFB and BP-1,4-DTFB were simulated by using the Materials Studio software, based on the attachment energy theory.

Calculation: For epitaxial growth of two crystalline materials, the lattice mismatch is defined as (a b)/a, where $\mathrm{a}$ and $\mathrm{b}$ are the corresponding lattice spacings of the underlying template and second growth layer, respectively. Thus, the lattice mismatch between the (110) plane of BP $\left(\mathrm{d}_{\mathrm{BP}, 110}=8.296 \AA\right)$ and the (011) plane of BP-1,3-DTFB ( $\left.\mathrm{d}_{\mathrm{BP}-1,3-\mathrm{DTFB}, 022}=1 / 2 \mathrm{~d}_{\mathrm{BP}-1,3-\mathrm{DTFB}, 011}=7.807 \AA\right)$ is estimated to be $5.9 \%$. While the lattice mismatch between the (110) plane of BP $\left(\mathrm{d}_{\mathrm{BP}, 110}=8.296 \AA\right)$ and the (101) plane of BP-1,4-DTFB ( $\left.\mathrm{d}_{\mathrm{BP}-1,4-\mathrm{DTFB}, 101}=7.956 \AA\right)$ is estimated to be $4.1 \%$. 


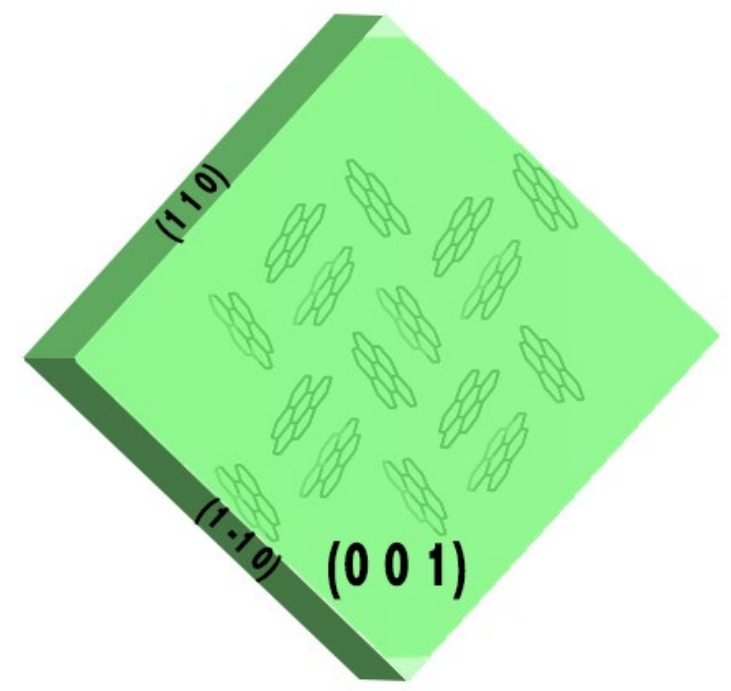

Figure S1. The simulated morphology of BP crystal based on growth morphology method.

Table S1. Summary of crystallographic data of BP, BP-1,3-DTFB, and BP-1,4-DTFB.

\begin{tabular}{llll}
\hline Molecular crystals & BP & BP-1,3-DTFB & BP-1,4-DTFB \\
\hline Cell lengths $(\AA)$ & $\mathrm{a}=11.72$ & $\mathrm{a}=7.02$ & $\mathrm{a}=9.15$ \\
& $\mathrm{~b}=11.88$ & $\mathrm{~b}=33.94$ & $\mathrm{~b}=7.03$ \\
& $\mathrm{c}=9.89$ & $\mathrm{c}=17.5^{\circ}$ & $\mathrm{c}=16.44$ \\
Cell angles & $\alpha=90^{\circ}$ & $\alpha=90^{\circ}$ & $\alpha=90^{\circ}$ \\
& $\beta=98.5^{\circ}$ & $\beta=90.1^{\circ}$ & $\beta=90.62^{\circ}$ \\
& $\gamma=90^{\circ}$ & $\gamma=90^{\circ}$ & $\gamma=90^{\circ}$ \\
Cell volume $(\AA 3)$ & 1361.9 & 4191.7 & 1057.0 \\
\hline
\end{tabular}


a

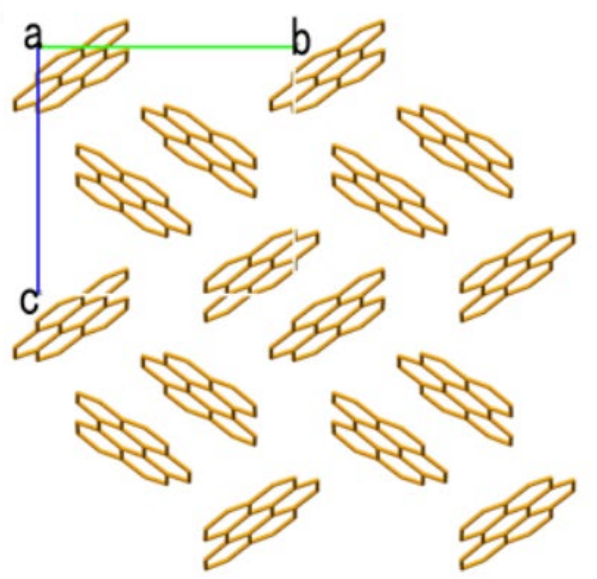

b

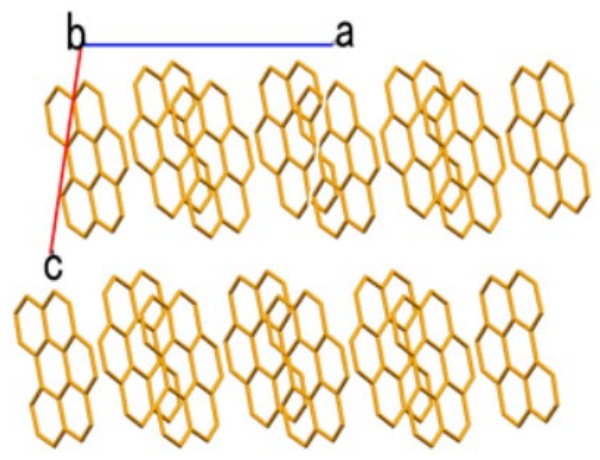

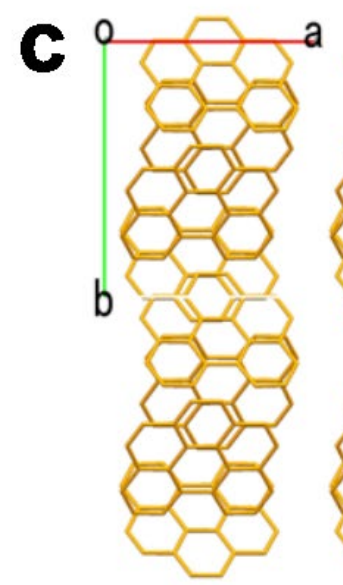

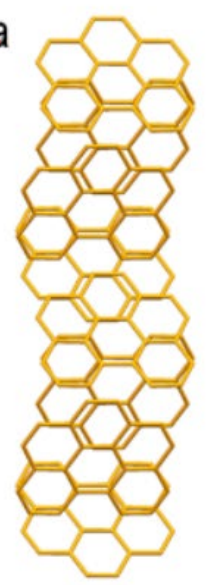

d

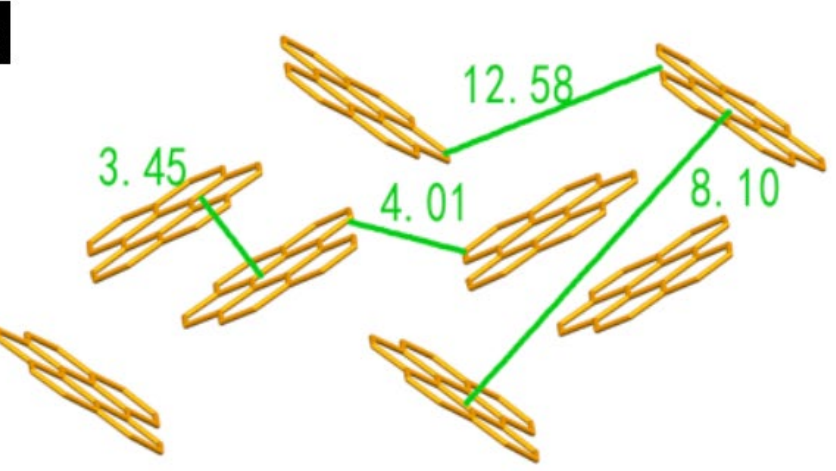

Figure S2. Molecular packing modes of BP crystal perpendicular to the (a) $a-$-, (b) $b$-, and (c) $c$-axis. (d) Intermolecular distances were measured in BP crystal. 


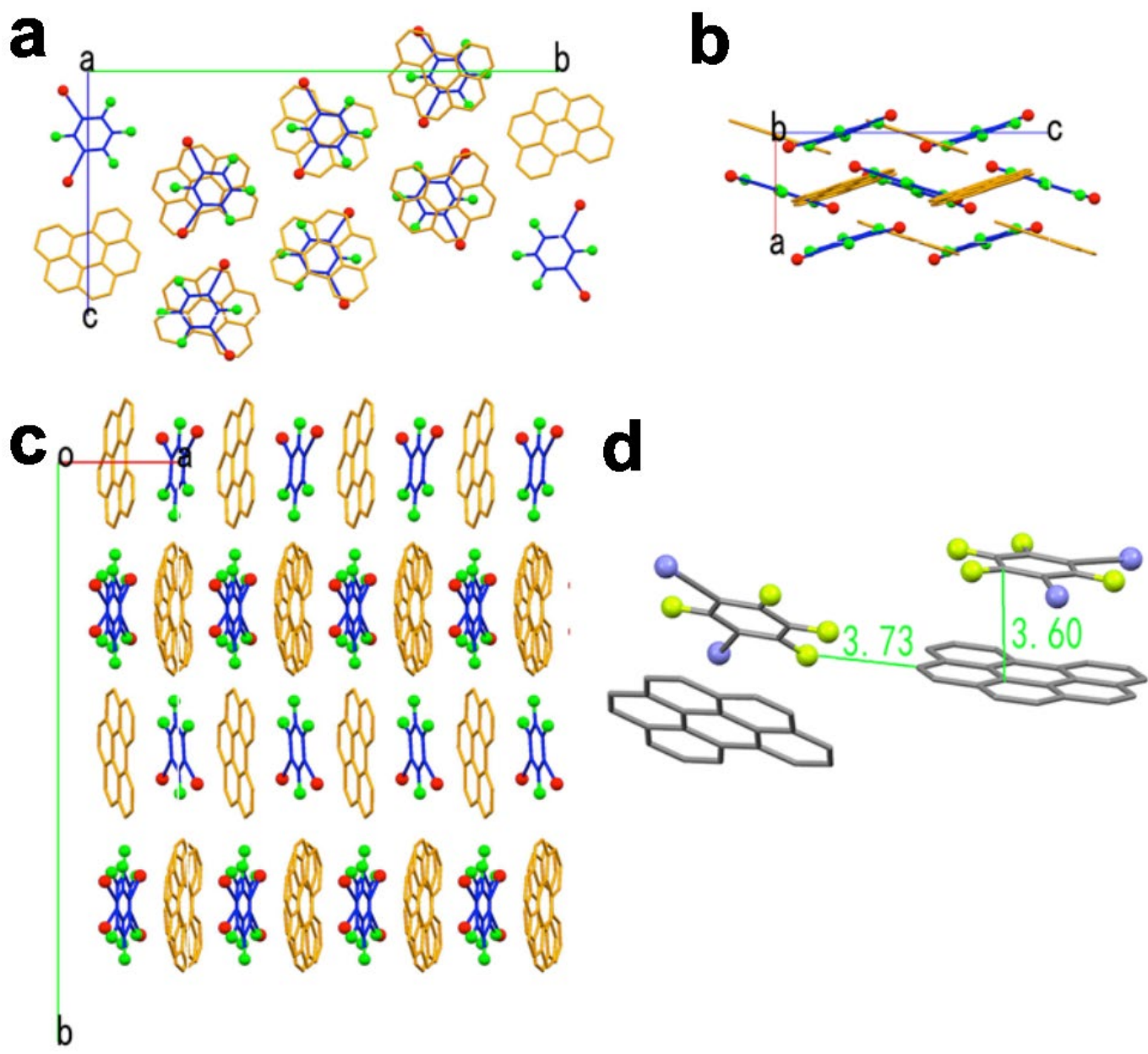

Figure S3. Molecular packing modes of BP-1,3-DTFB cocrystal perpendicular to the (a) $a$-, (b) $b$-, and (c) c-axis. (d) Intermolecular distances were measured in BP-1,3-DTFB cocrystal. 
a

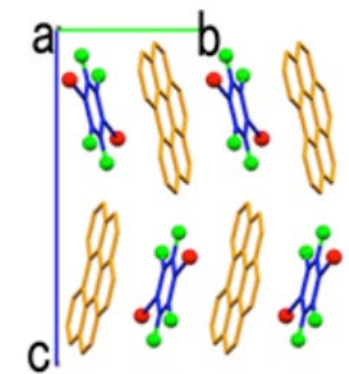

yo $y$ if

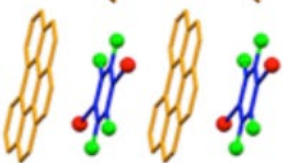

C

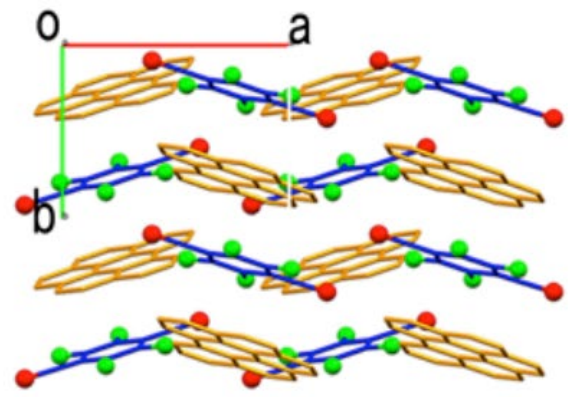

b

0
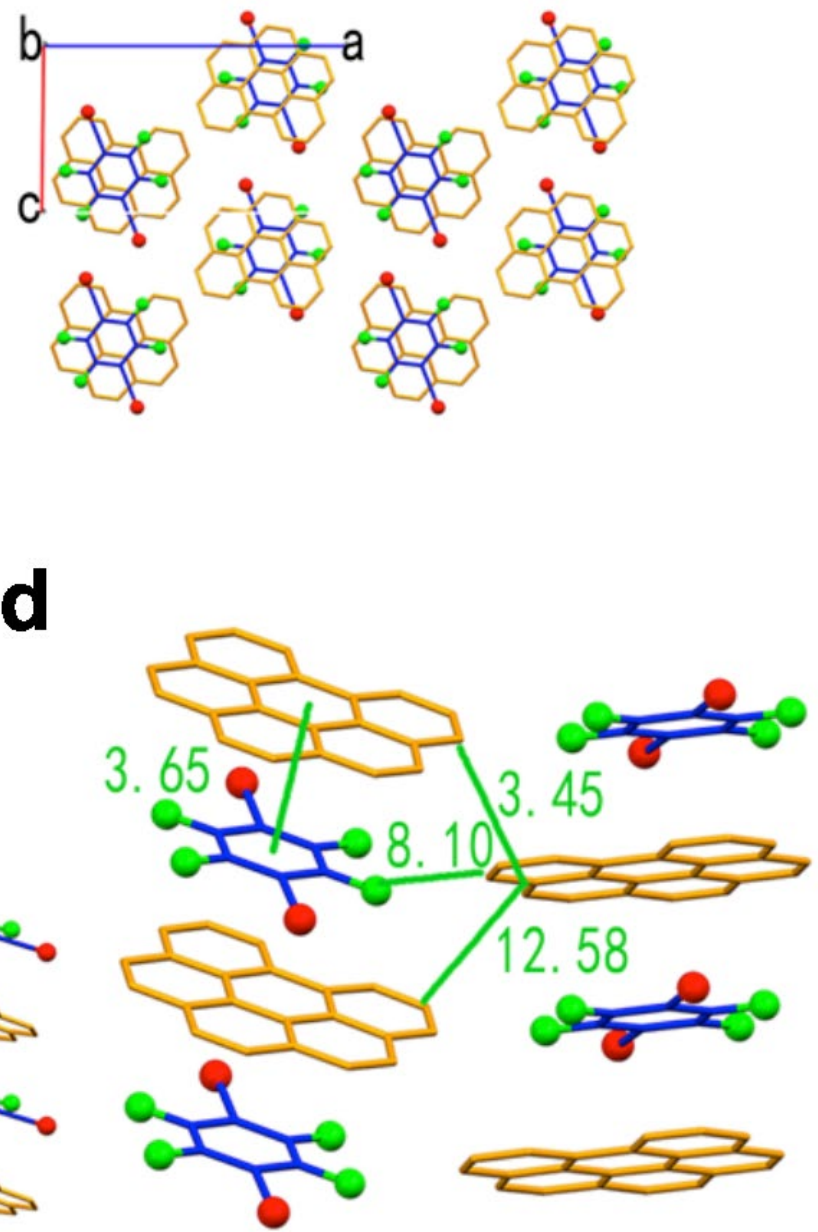

Figure S4. Molecular packing modes of BP-1,4-DTFB cocrystal perpendicular to the (a) $a-$, (b) $b$-, and (c) $c$-axis. (d) Intermolecular distances were measured in BP-1,4-DTFB cocrystal.

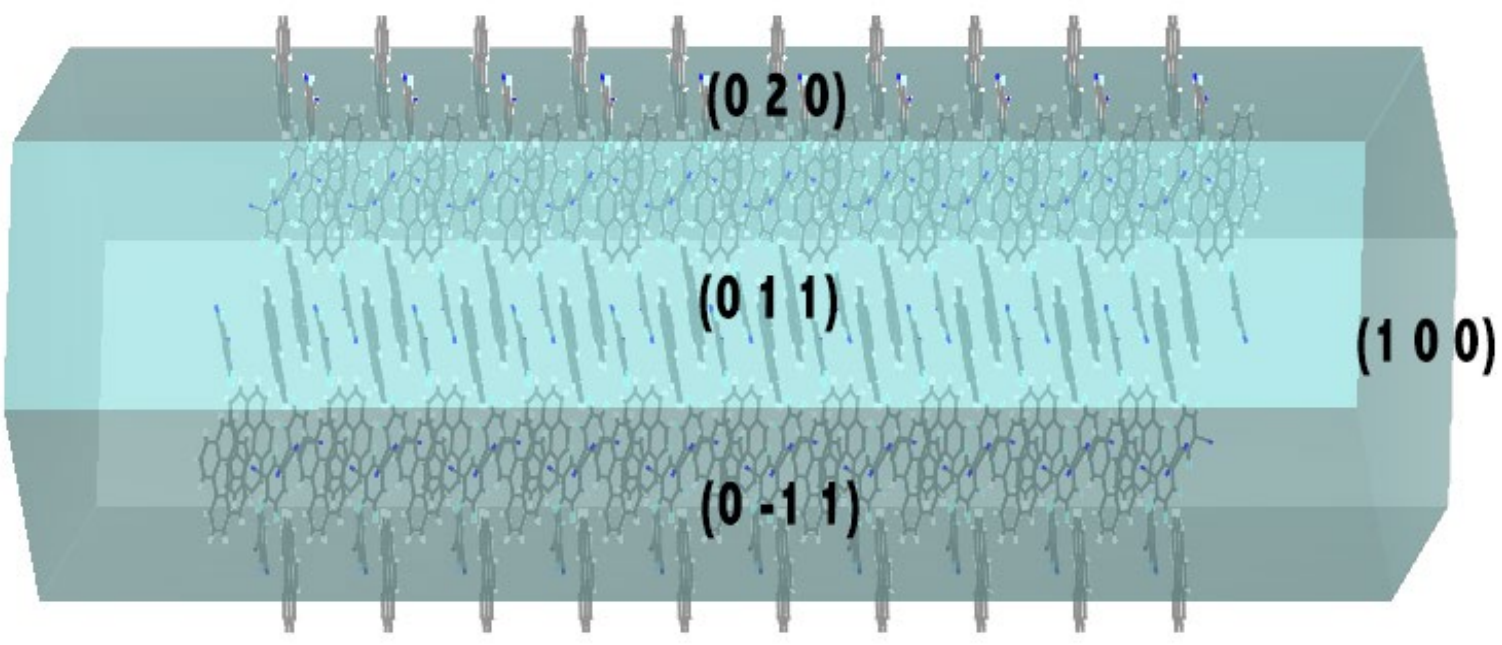

Figure S5. The simulated morphology of BP-1,3-DTFB cocrystal based on growth morphology method. 

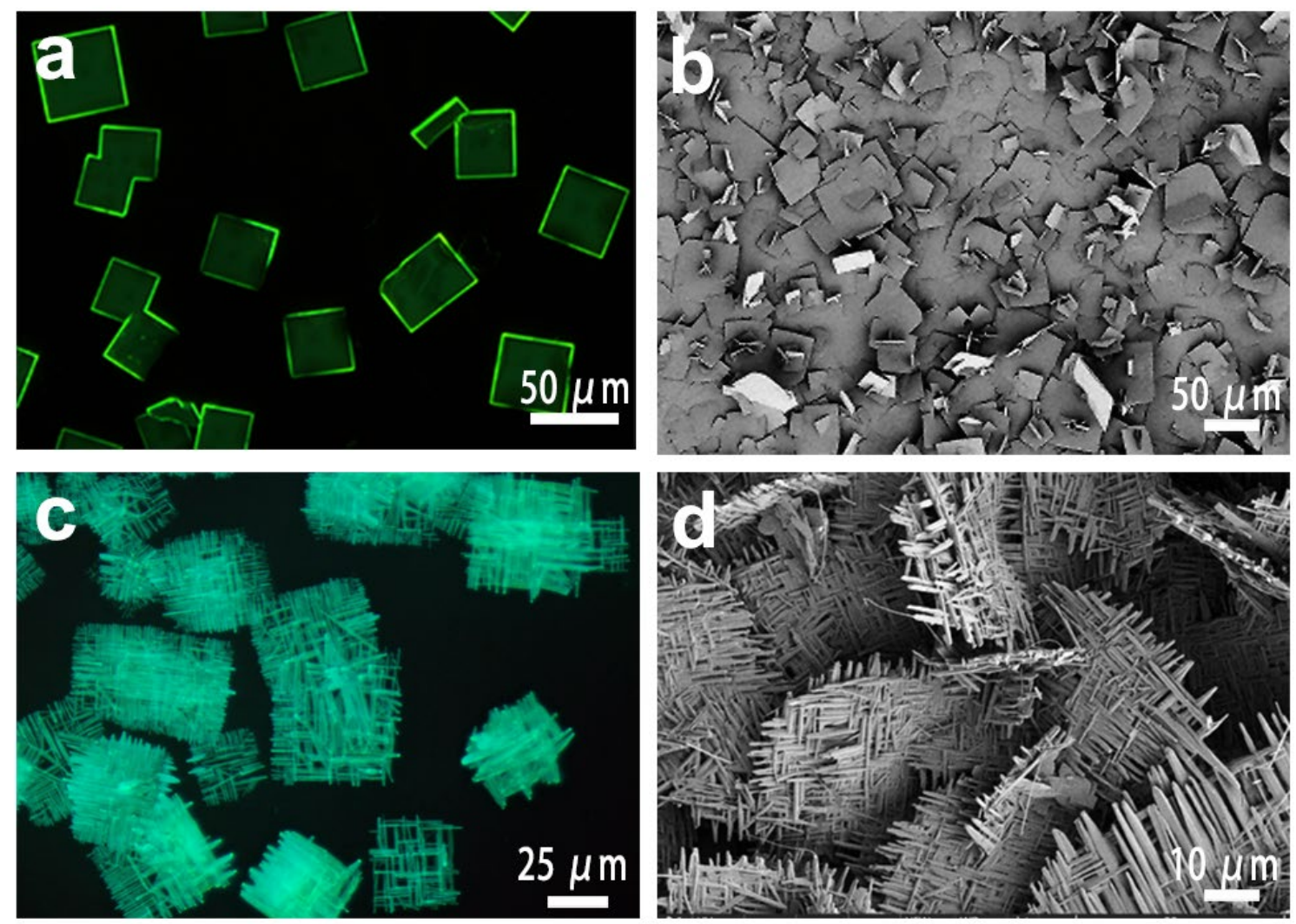

Figure S6. (a) FM and (b) SEM images of small-sized BP sheets with a width of around $50 \mu \mathrm{m}$. (c) FM and (d) SEM images small-sized BP-1,3-DTFB nanorod meshes.
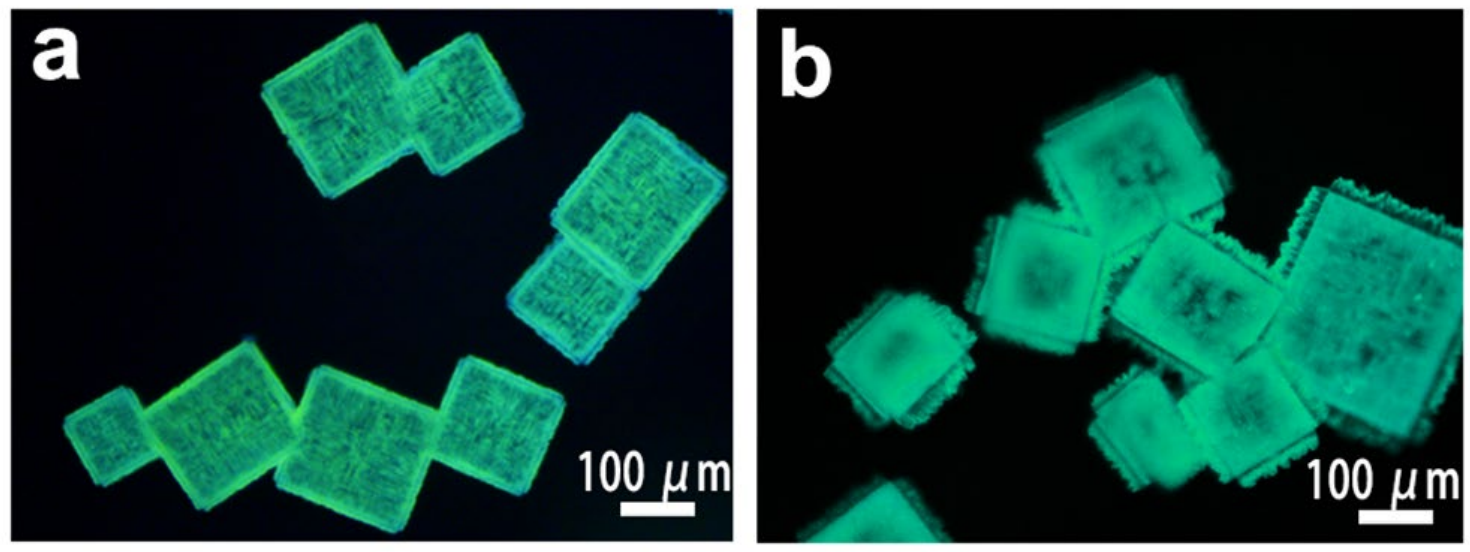

Figure S7. FM images of partially transformed BP microsheets upon (a) immersing them into 1,3DTFB solution in IPA with low concentration $\left(\mathrm{C}_{\mathrm{DTFB}}=50 \mathrm{mM}\right)$ or $(\mathrm{b})$ rapid evaporation of the suspension of BP microsheets dispersed in 1,3-DTFB solution in IPA $\left(\mathrm{C}_{\mathrm{DTFB}}=100 \mathrm{mM}\right)$. 

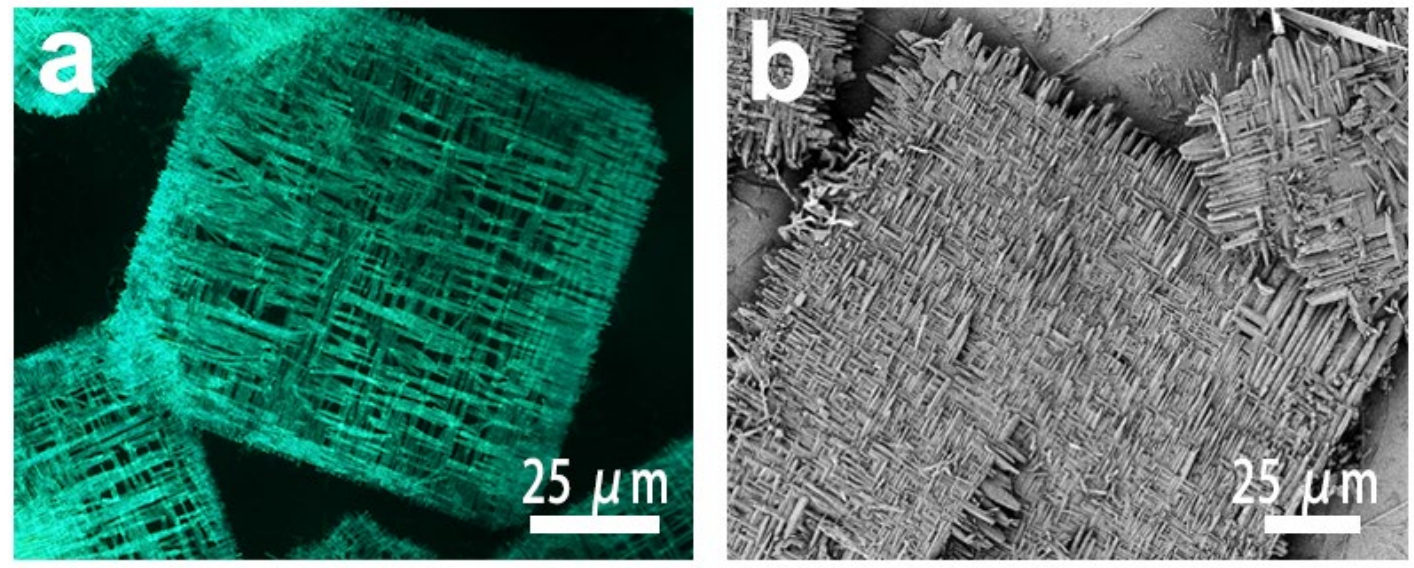

Figure S8. (a) FM and (b) SEM images of multilayer BP-1,3-DTFB nanorod meshes.
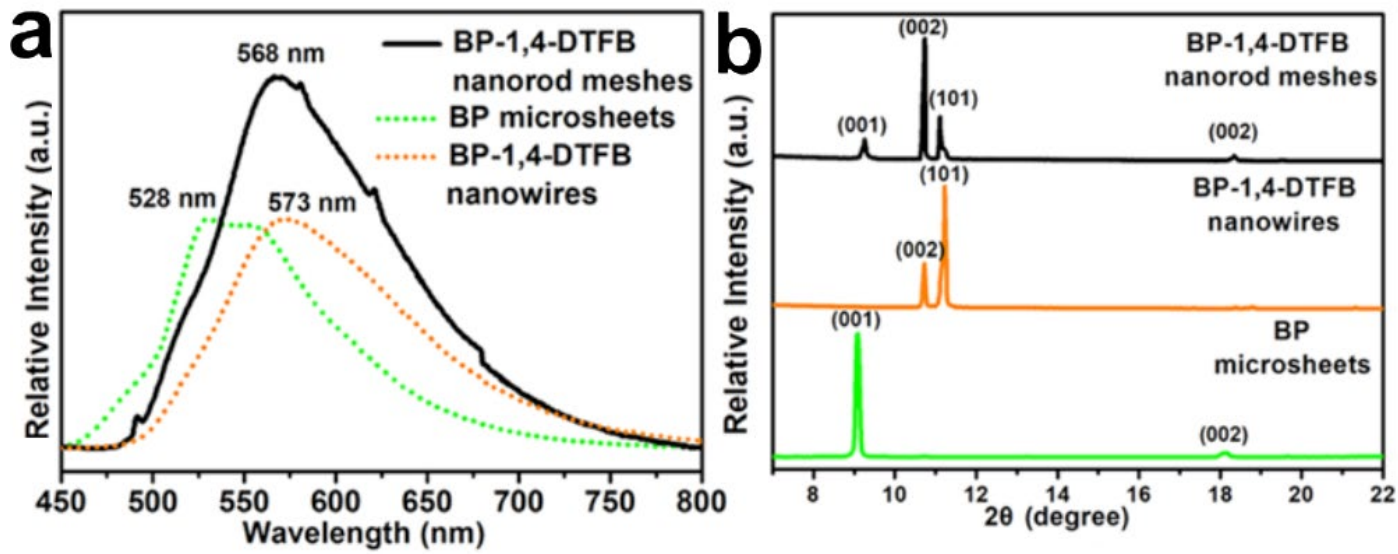

Figure S9. (a) PL spectra of BP microsheets, BP-1,4-DTFB microwires, and BP-1,4-DTFB nanorod meshes. (b) The corresponding PXRD patterns. 

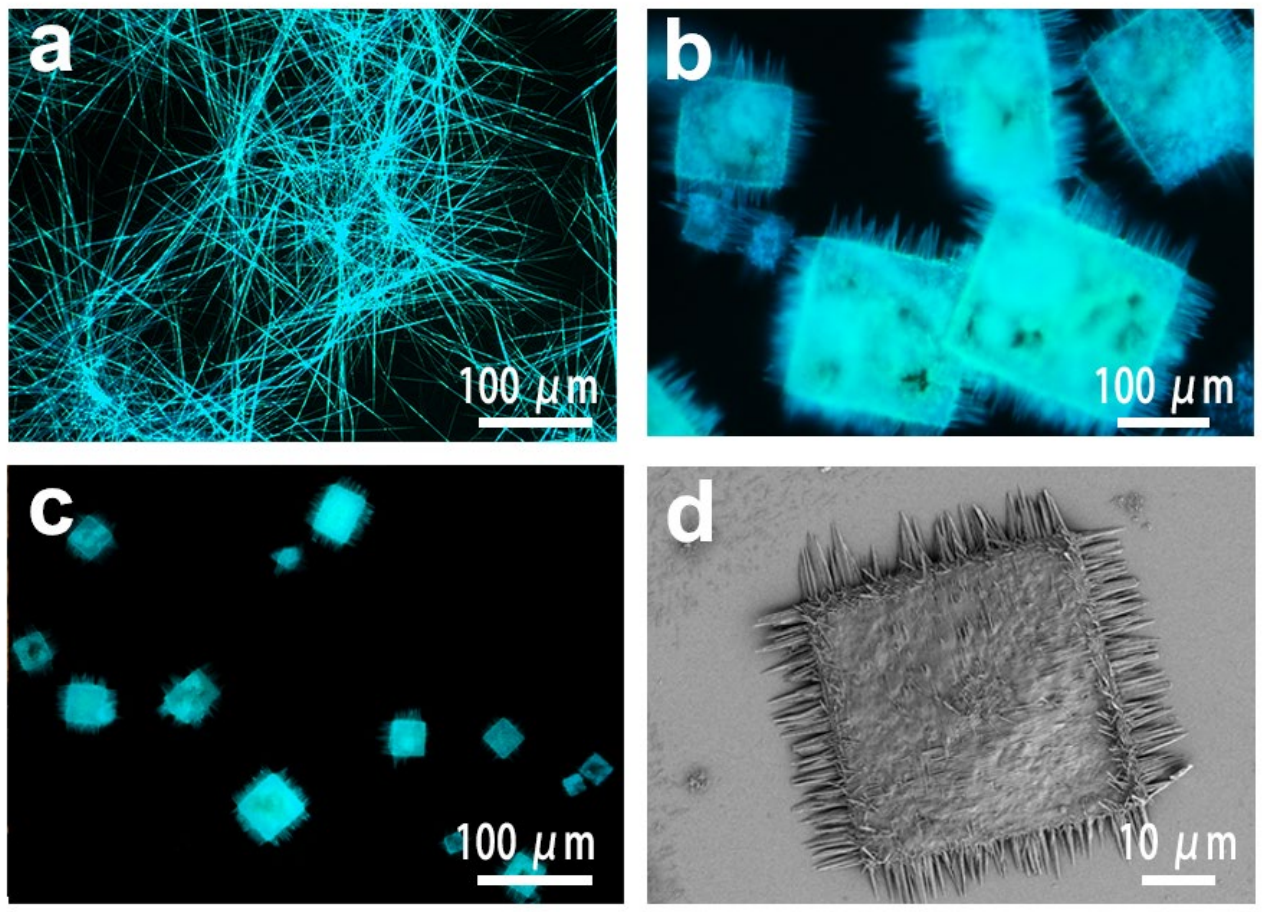

Figure S10. FM images of (a) BP-octafluoronaphthalene (BP-OFN) cocrystal microwires, (b, c) partially transformed BP-OFN nanorod meshes, and (d) SEM image of a single partially transformed BP-OFN nanorod mesh.
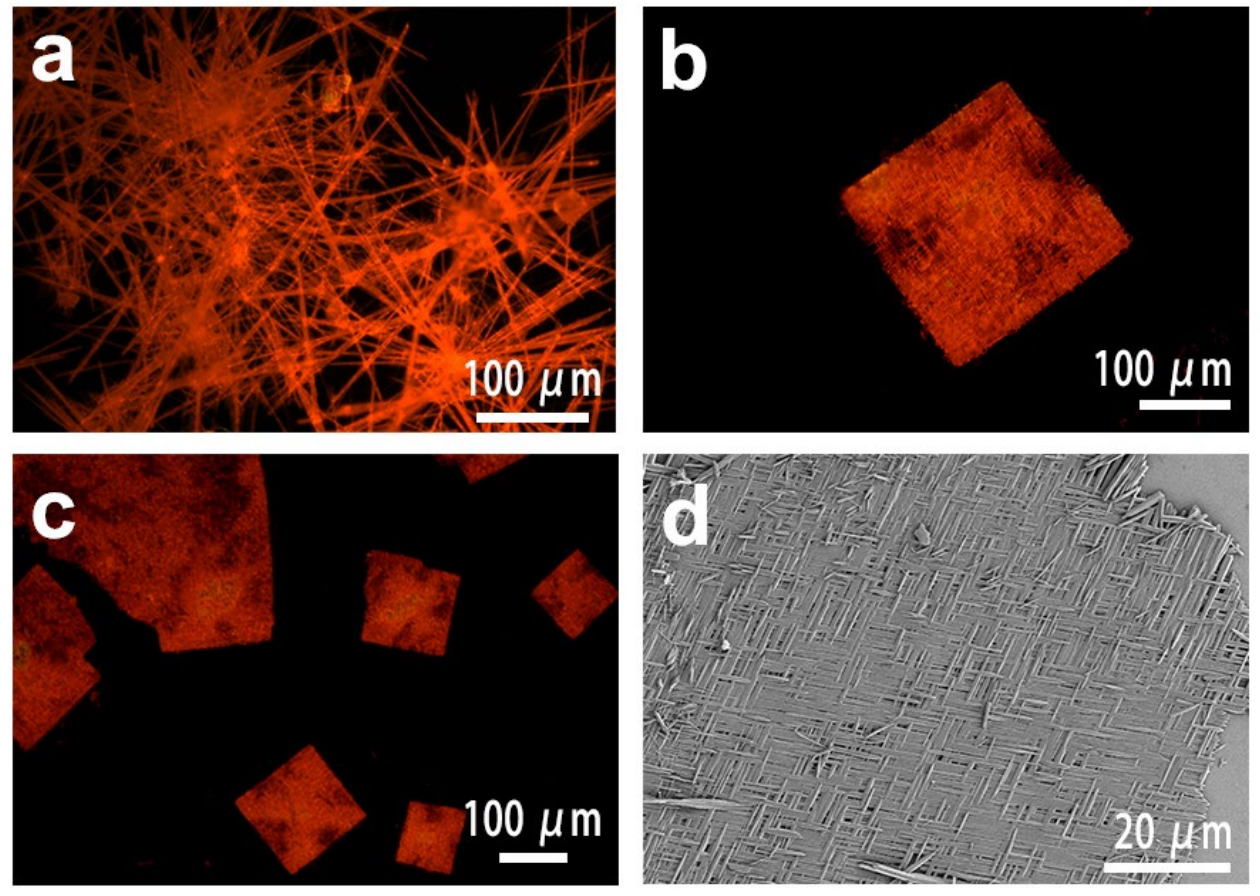

Figure S11. FM images of (a) BP-TCNB cocrystal microwires, (b, c) BP-TCNB nanorod meshes, and (d) SEM image of a single BP-TCNB nanorod mesh. 


\section{$\left(\begin{array}{lll}1 & 0 & 0\end{array}\right)$ \\ $\left(\begin{array}{llll}1 & 0 & 1\end{array}\right)$ \\ $\left(\begin{array}{lll}0 & 0 & 1\end{array}\right)$}

Figure S12. The simulated morphology of BP-1,4-DTFB cocrystal based on growth morphology method.

Table S2. Calculated attachment energies of different crystal facets of BP crystal.

\begin{tabular}{lcccc}
\hline hkl & Multiplicity & $\mathbf{d}_{\text {hkl }} / \AA$ & $\mathbf{E}_{\text {att }}($ Total $) / \mathbf{k c a l} \cdot \mathbf{m o l} \mathbf{l}^{\mathbf{1}}$ & \% Total facet area \\
\hline (oo1) & 2 & 9.78 & -24.94 & 43.03 \\
$(110)$ & 4 & 8.30 & -37.86 & 56.71 \\
(o11) & 4 & 7.55 & -51.38 & 0.26 \\
\hline
\end{tabular}

Table S3. Calculated attachment energies of different crystal facets of BP-1,3-DTFB cocrystal.

\begin{tabular}{|c|c|c|c|c|}
\hline hkl & Multiplicity & $\mathrm{d}_{\mathrm{hkl}} / \AA$ & 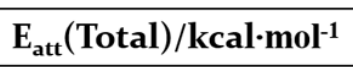 & \% Total facet area \\
\hline (020) & 2 & 16.97 & -70.47 & 31.31 \\
\hline (o11) & 4 & 15.61 & -73.76 & 53.46 \\
\hline (100) & 2 & 7.02 & -202.06 & 14.96 \\
\hline
\end{tabular}

Table S4. Calculated attachment energies of different crystal facets of BP-1,4-DTFB cocrystal.

\begin{tabular}{lcccc}
\hline hkl & Multiplicity & $\mathbf{d}_{\text {hkl }} / \mathbf{A}$ & $\mathbf{E}_{\text {att }}($ Total $) / \mathbf{k c a l}^{-\mathbf{m o l}^{\mathbf{1}}}$ & \% Total facet area \\
\hline (oo1) & 2 & 16.44 & $-17 \cdot 74$ & 49.41 \\
$(100)$ & 2 & 9.15 & -36.24 & 15.92 \\
$(10-1)$ & 2 & 8.03 & -35.69 & 8.99 \\
$(101)$ & 2 & 7.96 & -37.14 & 5.62 \\
$(\mathrm{ol1})$ & 4 & 6.46 & -55.77 & 18.71 \\
\hline
\end{tabular}

